\title{
EFFECT OF FIBER SOURCE AND LEVEL WITH / OR WITHOUT ENZYME SUPPLEMENTATION ON GROWTH PERFOPMANCE OF BROILER CHICKS
}

\author{
Ahmed E.M. Eliwah", S.S. Aboul Ela, A.I. Attia and I. E. Ismail \\ Poult. Dept., Fac. Agric., Zagazig Univ., Egypt
}

\begin{abstract}
A $2 \times 3 \times 2$ factorial design experiment was performed including two sources of fiber (corn cobs and wheat straw), three levels of fiber (3, 6 and $9 \%$ ) and two levels of enzyme (without or $250 \mathrm{~g} / \mathrm{kg}$ diet) through the experimental period (1-5 weeks of age). A total number of 360 unsexed one week old Cobb broiler chicks were randomly distributed into 12 treatment groups of 30 chicks each with 3 replicates (10 chicks each). Growth performance (live body weight, body weight gain, feed intake and feed conversion ratio) were studied through out the period from 1- 5 weeks of age. Results obtained indicated, significant $(\mathrm{P}<0.01)$ improvement in most traits of growth performance (live body weight, body weight gain and feed conversion ratio) in groups fed corn cobs diet through all the experimental periods studied comparatively with those received wheat straw diets, except, feed intake, in which increased $(\mathrm{P}<0.01)$ in groups fed wheat straw diets. However, significant $(\mathrm{P}<0.01)$ improvement in all traits of growth performance was observed for chicks fed diets containing $3 \%$ crude fiber level when compared with those fed diets containing 6 or $9 \%$ crude fiber levels. Addition of enzyme to the diets of broiler chicks significantly $(\mathrm{P}<0.01)$ improved live body weight, body weight gain and feed conversion ratio, while feed intake was decreased through out the experimental periods studied due to enzyme supplementation to broiler chicks diet. Chicks fed corn cobs diet at $3 \%$ crude fiber level with enzyme supplementation recorded the highest values of growth performance of broiler chicks while the lowest values were obtained by chicks fed wheat straw diets at $9 \%$ crude fiber level and without enzyme supplementation. It could be concluded that, supplementation of enzyme to diet contained corn cobs at $3 \%$ crude fiber would be suitable of broiler chicks through 1- 5 weeks of age for obtaining high growth performance.
\end{abstract}

Key words: Growth performance, corn cobs, wheat straw, enzyme, crude fiber.

\section{INTRODUCTION}

Fiber source of natural plants could be used as a possible nutritional and economical alternative source to supplement scavenging intake of free-range chickens, however there are only a few reports in the literature which evaluate utilization of natural plants fiber source for improving poultry health and production. McDonald and Whitesides (2002) defined fiber as a term that refers to cell walls of plant tissue that mostly consist of lignin, cellulose as well as hemicelluloses. Further, it as the composition of plant cell that is resistant against enzymes in the small intestine. Moreover, from the chemical point of view, fiber is illustrated as non-starch polysaccharides. According to McNab and Boormann (2002), non-starch polysaccharides (NSP) could be divided into two types of (soluble and insoluble). Based on a research conducted by Branton et al.(1997) a noncontagious disease takes place in poultry every time that diet enriches of insoluble NSP results in more risk of necrotic enteritis. This is because of an increasing microbial fermentation in the intestine. The two terms of "crude fiber" and

\footnotetext{
*Corresponding author: Tel. : +201201515906

E-mail address: aeliwah@yahoo.com
} 
"roughage" are mostly applied synonymously in animal nutrition. Crude fiber refers to the structural carbohydrates made of cellulose, hemicelluloses and lignin in the plant cell wall while the composition of crude fiber in each single plant is different between plants.

The latest researches precisely recognize the percentage of crude fiber for poultry; crude fiber could be in a range from 3 to $4 \%$ for a greater period while it could be applied by $5 \%$ for layers. Generally, poultry-feed manufacturers and poultry producers believe that fiber content must be kept below 7\% in poultry feed. Fiber is viewed negative as it declines production as well as chicken growth, it looks to decrease the effectiveness of feed utilization.

The effects of dietary fiber in chickens are not well understood but it is known to contribute little to the nutrition (Larrea and Garcia, 1963). However, fiber influences the utilization of other nutrients in the food through changes in gut transit time, digestion, absorption and therefore, food intake.

The best level of dietary fiber, which achieves acceptable performance, varied from study to another (Hill and Dansky, 1954 ; Abdelsamie and Yadiwilo, 1981).

Abbas (1992) reported that chick, feed mixtures must contains a sufficient concertation of required nutrients of good digestibility to achieve the best feed efficiency. The same author added that the dietary fiber up to $7 \%$ has no effect on performance of chick, while some adverse effects were observed at the level of $9 \%$ although in some cases especially with feed energy no differences were found between all treatments. The increment of fiber in the diet up to $7 \%$ was accompanied by increases in feed conversion with the advancement of age. This demonstrated the capacity of chick to accommodate large volumes of feed in an attempt to maintain a static level of nutrient intake in order to satisfy their requirements.

Traditionally, fiber represents the indigestible component in poultry diets because birds do not digest cellulose (Tasaki and Kibe, 1959). Janssen and Carre (1985) showed that a strong negative correlation between crude fiber content of the diet and protein and fat digestibility in broilers and concluded that lowcrude fiber diets improve poultry performance. However, the inclusion of moderate amounts of fiber might benefit digestive physiology in poultry (Hedemann et al., 2006; Mateos et al., 2006). In fact, Hetland et al. (2003) reported that moderate levels of insoluble fiber in the diet increased the ilea digestibility of starch. Recently, Gonzalez-Alvarado et al. (2007) demonstrated that the inclusion of $3 \%$ oat hulls or soy hulls improved performance of broilers from 1 to 21 days of age and nutrient digestibility at 18 days of age.

Addition of enzymes such as amylase and xylanase are useful in the utilization of the non-starch polysaccharide component of the diet ingredients. Others, such as proteases supplementation may enhance the utilization of dietary protein. Therefore, enzymes supplementation increase the effectiveness of nutrient utilization resulting in improved performance (Acamovic, 2001).

Many enzymes have been found to be beneficial when added to poultry diets containing carbohydrate or protein source. This Avizyme product which contains amylase (improves corn starch utilization), xylanase (reduces viscosity and breaks down cereal cell walls), and protease (targets soybean meal antinutritional factors and storage proteins). Avizyme may also be effective in improving energy utilization in corn-soy diets (Michael, 2002). These data suggested a slight improvement in protein and amino acid utilization. In addition the Avizyme destroys anti-nutritional factors and increases the digestibility of indigestible nutrients. Thus the utilization of enzyme systems saves energy and improves amino acid digestibility (Lyons, 1995; Silversides and Bedford, 1999).

However the present study was conducted to evaluate the source and level of dietary fiber at which the chicks could tolerate without adverse effect on its performance using corn cobs and wheat straw as source of fiber with or without enzyme supplementation. 


\section{MATERIALS AND METHODS}

The experimental work was carried out at a private farm, near to Zagazig city, Sharkiya Governorate, Egypt, during the period from 15 April, 2014 to 1 June, 2014.

This work was designed to investigate the effect of source and level of dietary fiber with or without enzyme supplementation on growth performance of Cobb broiler chicks. A $2 \times 3 \times 2$ factorial design experiment was performed including two sources of fiber (corn cobs and wheat straw), three levels of fiber (3, 6 and 9\%) and two levels of enzyme (without or $250 \mathrm{~g} / \mathrm{kg}$ diet) through the experimental period (1-5 week of age). A total number of 360 unsexed one week old Cobb broiler chicks were randomly distributed into 12 treatment groups of 30 chicks each with 3 replicates, (10 chicks each). Chicks of all experimental groups had nearly the same initial average live body weight and were not statistically different. Six iso coloric-iso nitrogenous diets were formulated to cover the nutrient requirements of broiler chicks during strater (1-3 weeks of age) and grower/finisher (3-5 weeks of age) periods. Diets contained three levels of crude fiber (3, 6 and 9\%) from corn cobs and the same levels from wheat straw, the composition and chemical analysis of the starter and grower/ finisher experimental diets are presented in Tables 2 and 3.

Diets were supplemented or not with kemzyme ( $250 \mathrm{~g} / \mathrm{kg}$ diet). Kemzyme used in this study is a natural multi-enzyme fed supplement containing : Alpha-amylase, Beta-gluconases, proteases and cellulose. Enzyme was purchased from Multivita Company, six ${ }^{\text {th }}$ of October, Egypt.

Birds were allocated on floor and kept under similar conditions of management. Artificial light source was used, giving a total of 23 hours of light per day throughout the experimental period. Gas heaters were used to provide chicks with needed heat for brooding. Room temperature was about $32^{\circ} \mathrm{C}$ for the first three days and then decreased $0.3^{\circ} \mathrm{C}$ daily until $24^{\circ} \mathrm{C}$, reaching thereafter to the normal temperature. Electric fans were used to achieve a regular circulation of air up to 35 days of chick's age in all treatment groups. Chicks were provided with feed and water for ad-libitum consumption. The experimental period was extended 5 weeks.

Chicks were individually weighed at 1,3 and 5 weeks of age. Also, body weight gain was calculated. Feed intake data were weekly recorded on a replicate basis during the experimental period, consequently, feed conversion was estimated (g feed/g gain).

Data were statistically analyzed on a $2 \times 3 \times 2$ factorial design basis according to Snedecor and Cochran (1982) using SPSS ${ }^{\circledR}$ software statistical analysis program (SPSS, 1999), by adopting the following model:

$\mathrm{Y}_{\mathrm{ijk} l}=\mu+\mathrm{S}_{\mathrm{i}}+\mathrm{L}_{\mathrm{j}}+\mathrm{E}_{\mathrm{k}}+\mathrm{SL}_{\mathrm{ij}}+\mathrm{SE}_{\mathrm{ik}}+\mathrm{LE}_{\mathrm{jk}}+\mathrm{SLE}_{\mathrm{ijk}}$ $+\mathrm{e}_{\mathrm{ijkl}}$

Where:

$\mathrm{Y}_{\mathrm{ijkl}}=$ an observation,

$\mu=$ the overall mean,

$\mathrm{S}_{\mathrm{i}}=$ effect of fiber source,

$\mathrm{L}_{\mathrm{j}}=$ effect of fiber level,

$E_{k}=$ effect of enzyme supplementation

$\mathrm{SL}_{\mathrm{ij}}=$ effect of interaction between fiber source and level,

$\mathrm{SE}_{\mathrm{ik}}=$ effect of interaction between fiber source and enzyme,

$\mathrm{LE}_{\mathrm{jk}}=$ effect of interaction between fiber level and enzyme,

$\mathrm{SLE}_{\mathrm{ijk}}=$ effect of interaction among fiber source, fiber level and enzyme,

$\mathrm{e}_{\mathrm{ijkl}}=$ experimental random error.

Duncan's new multiple range test (Duncan, 1955) was used for comparison among significant means.

\section{RESULTS AND DISCUSSION}

\section{Growth Performance}

\section{Effect of fiber source}

Results in Tables 4 and 5 reveale that, live body weight at 3 and 5 weeks of age and body weight gain during starter ( 1 - 3 weeks of age), 
Table 1. Chemical composition of feed stuffs

\begin{tabular}{|c|c|c|}
\hline Feed stuff & Corn cobs & Wheat straw \\
\hline Dry matter (\%) & 90.0 & 90.0 \\
\hline Crude protein (\%) & 4.0 & 3.2 \\
\hline Ether extract (\%) & 1.0 & 1.5 \\
\hline \multicolumn{3}{|l|}{ Fiber } \\
\hline Crude fiber (\%) & 29.2 & 42.1 \\
\hline Acid detergent fiber (\%) & 33.0 & 55.0 \\
\hline Neutral detergent fiber (\%) & 57.0 & 80.0 \\
\hline Legnin (\%) & 9.4 & 9.0 \\
\hline Cellulose (\%) & 21.3 & 36.0 \\
\hline Ash (\%) & 6.1 & 8.0 \\
\hline \multicolumn{3}{|l|}{ Macroelements } \\
\hline Calcium & 0.52 & 0.16 \\
\hline Total phosphorous & 0.20 & 0.05 \\
\hline Available phosphorous & - & - \\
\hline \multicolumn{3}{|l|}{ Amino Acids } \\
\hline Lysine (\%) & - & - \\
\hline Methionine (\%) & - & - \\
\hline Methionine + Cystine (\%) & - & - \\
\hline Threonine (\%) & - & - \\
\hline Tryptophane (\%) & - & - \\
\hline \multicolumn{3}{|l|}{ Energy Values } \\
\hline Gross Energy K cal/kg & 4000 & 3800 \\
\hline Metabolizable energy poultry (K cal/kg) & - & - \\
\hline Digestible energy for rabbits (K cal/kg) & - & - \\
\hline
\end{tabular}


Table 2. Composition and chemical analysis of the experimental starter diets (1-3 weeks of age).

\begin{tabular}{|c|c|c|c|c|c|c|}
\hline \multirow[t]{2}{*}{ Ingredient } & \multicolumn{3}{|c|}{ Corn cobs } & \multicolumn{3}{|c|}{ Wheat straw } \\
\hline & 3 & 6 & 9 & 3 & 6 & 9 \\
\hline Yellow corn & 50.1 & 46.1 & 37.5 & 50.5 & 44.3 & 41.4 \\
\hline Soybean meal 44\% & 30 & 29.5 & 27.2 & 30.3 & 29.7 & 29.1 \\
\hline Corn gluten $62 \%$ & 7 & 8 & 9.5 & 7 & 7.6 & 8.7 \\
\hline Corn cobs & 3 & 6 & 9 & 0 & 0 & 0 \\
\hline Wheat straw & 0 & 0 & 0 & 3 & 6 & 9 \\
\hline Cotton seed oil & 4.2 & 6 & 6.5 & 4.8 & 5.3 & 7.4 \\
\hline Salt & 0.3 & 0.3 & 0.3 & 0.3 & 0.3 & 0.3 \\
\hline Premix $^{1}$ & 0.4 & 0.4 & 0.4 & 0.4 & 0.4 & 0.4 \\
\hline Wheat bran & 1.3 & 0 & 6 & 0 & 2.8 & 0 \\
\hline Anti-toxins & 0.1 & 0.1 & 0.1 & 0.1 & 0.1 & 0.1 \\
\hline Limestone & 1.5 & 1.4 & 1.4 & 1.5 & 1.4 & 1.4 \\
\hline Di-calcium phosphate & 1.5 & 1.6 & 1.5 & 1.5 & 1.5 & 1.6 \\
\hline L-lysine & 0.4 & 0.4 & 0.4 & 0.4 & 0.4 & 0.4 \\
\hline Dl-Methionine & 0.2 & 0.2 & 0.2 & 0.2 & 0.2 & 0.2 \\
\hline Total & 100 & 100 & 100 & 100 & 100 & 100 \\
\hline \multicolumn{7}{|l|}{ Chemical analysis } \\
\hline \multicolumn{7}{|l|}{ a- Calculated ${ }^{2}$} \\
\hline ME, Kcal /Kg & 3075 & 3073 & 3086 & 3077 & 3081 & 3072 \\
\hline Crude protein (\%) & 22.70 & 22.74 & 22.75 & 22.72 & 22.78 & 22.74 \\
\hline Calcium (\%) & 1.03 & 1.01 & 1.03 & 1.04 & 1.01 & 1.02 \\
\hline Phosphorous (\%) & 0.42 & 0.43 & 0.43 & 0.42 & 0.42 & 0.43 \\
\hline$M+C(\%)$ & 0.98 & 0.98 & 1.00 & 0.98 & 0.98 & 0.98 \\
\hline Linoleic (\%) & 1.24 & 1.13 & 1.04 & 1.23 & 1.14 & 1.03 \\
\hline Crude fiber (\%) & 3 & 6 & 9 & 3 & 6 & 9 \\
\hline \multicolumn{7}{|l|}{ b- Determined ${ }^{3}$} \\
\hline Crude protein (\%) & 21.89 & 22.01 & 22.76 & 21.98 & 22.87 & 22.56 \\
\hline Crude fat (\%) & 3.50 & 2.95 & 2.87 & 3.00 & 2.86 & 2.67 \\
\hline Crude fiber (\%) & 3.21 & 6.13 & 9.22 & 3. 14 & 6.34 & 9.20 \\
\hline Ash (\%) & 4.82 & 5.43 & 5.01 & 5.27 & 5.12 & 4.72 \\
\hline
\end{tabular}

${ }^{\mathrm{T}}$ The vitamin premix supplied the following per kilogram of complete feed: vitamin A, 4,500 IU (retinyl acetate); cholecalciferol, 1,000 IU; vitamin E, 25IU (dl-a-tocopheryl acetate); vitamin B12,0.02 mg; menadione, $1.5 \mathrm{mg}$; riboflavin, $3 \mathrm{mg}$; thiamine, $1.5 \mathrm{mg}$; pantothenic acid, $5 \mathrm{mg}$; niacin, $20 \mathrm{mg}$; choline, $150 \mathrm{mg}$; folic acid, $0.5 \mathrm{mg}$; biotin, $0.5 \mathrm{mg}$; pyridoxine, $2.5 \mathrm{mg}$.The mineral premix supplied the following per kilogram of complete feed: manganese (MnSO4. $\mathrm{H} 2 \mathrm{O}$ ), $60 \mathrm{~g}$; zinc $(\mathrm{ZnO}), 40 \mathrm{mg}$; iron (FeSO4.7H2O), $80 \mathrm{mg}$; copper (CuSO4.5H2O), 8 mg; selenium (Na2SeO3), 0.2 mg; iodine (Iodized $\mathrm{NaCl}$ ), $0.8 \mathrm{mg}$; cobalt (CoCl2), $0.4 \mathrm{mg}$. 
${ }^{2}$ Calculated according to NRC (1994).

${ }^{3}$ Determined according to AOAC (2003).

Table 3. Composition and chemical analysis of the experimental grower/finisher diets (3-5 weeks of age).

\begin{tabular}{|c|c|c|c|c|c|c|}
\hline \multirow[t]{2}{*}{ Ingredient } & \multicolumn{3}{|c|}{ Corn cobs } & \multicolumn{3}{|c|}{ Wheat straw } \\
\hline & 3 & 6 & 9 & 3 & 6 & 9 \\
\hline Yellow corn & 55.4 & 50.5 & 45.6 & 57.3 & 52.3 & 49 \\
\hline Soybean meal $44 \%$ & 26.3 & 25.5 & 23.5 & 26 & 25.8 & 23.2 \\
\hline Corn gluten $62 \%$ & 4.8 & 5.6 & 7.2 & 5 & 5.6 & 7.7 \\
\hline Corn cobs & 3 & 6 & 9 & 0 & 0 & 0 \\
\hline Wheat straw & 0 & 0 & 0 & 3 & 6 & 9 \\
\hline Cotton seed oil & 5 & 5.3 & 6.5 & 4.6 & 6.2 & 7 \\
\hline Salt & 0.3 & 0.3 & 0.3 & 0.3 & 0.3 & 0.3 \\
\hline Premix $^{1}$ & 0.4 & 0.4 & 0.4 & 0.4 & 0.4 & 0.4 \\
\hline Wheat bran & 1.4 & 3 & 4.1 & 0 & 0 & 0 \\
\hline Anti-toxins & 0.1 & 0.1 & 0.1 & 0.1 & 0.1 & 0.1 \\
\hline Limestone & 1.3 & 1.3 & 1.3 & 1.3 & 1.3 & 1.3 \\
\hline Di-calcium phosphate & 1.4 & 1.4 & 1.4 & 1.4 & 1.4 & 1.4 \\
\hline L-lysine & 0.4 & 0.4 & 0.4 & 0.4 & 0.4 & 0.4 \\
\hline Dl-Methionine & 0.2 & 0.2 & 0.2 & 0.2 & 0.2 & 0.2 \\
\hline Total & 100 & 100 & 100 & 100 & 100 & 100 \\
\hline \multicolumn{7}{|l|}{ Chemical analysis } \\
\hline \multicolumn{7}{|l|}{ a- Calculated $^{2}$} \\
\hline ME, Kcal /Kg & 3157 & 3124.5 & 2164.6 & 3119 & 3126.1 & 3152.2 \\
\hline Crude protein (\%) & 20.10 & 20.15 & 20.07 & 20.07 & 20.08 & 20.07 \\
\hline Calcium (\%) & 0.93 & 0.94 & 0.93 & 0.91 & 0.92 & 0.91 \\
\hline Phosphorous (\%) & 0.39 & 0.39 & 0.38 & 0.39 & 0.39 & 0.38 \\
\hline$M+C(\%)$ & 0.89 & 0.89 & 0.90 & 0.89 & 0.88 & 0.90 \\
\hline Linoleic (\%) & 1.35 & 1.26 & 1.17 & 0.0 & 1.25 & 1.17 \\
\hline Crude fiber (\%) & 3 & 6 & 9 & 3 & 6 & 9 \\
\hline \multicolumn{7}{|l|}{ b- Determined ${ }^{3}$} \\
\hline Crude protein (\%) & 19.47 & 19.85 & 20.05 & 19.87 & 20.12 & 20.07 \\
\hline Crude fat (\%) & 3.85 & 3.25 & 3.16 & 3.30 & 3.15 & 2.94 \\
\hline Crude fiber (\%) & 3.16 & 6.18 & 9.52 & 3.32 & 6.26 & 9.17 \\
\hline Ash (\%) & 5.30 & 5.97 & 5.51 & 5.80 & 5.63 & 5.19 \\
\hline
\end{tabular}

${ }^{1}$ The vitamin premix supplied the following per kilogram of complete feed: vitamin A, 4,500 IU (retinyl acetate); cholecalciferol, 1,000 IU; vitamin E, 25IU (dl-a-tocopheryl acetate); vitamin B12,0.02 mg; menadione, 1.5 mg; riboflavin, $3 \mathrm{mg}$; thiamine, $1.5 \mathrm{mg}$; pantothenic acid, $5 \mathrm{mg}$; niacin, $20 \mathrm{mg}$; choline, $150 \mathrm{mg}$; folic acid, $0.5 \mathrm{mg}$; biotin, $0.5 \mathrm{mg}$; pyridoxine, $2.5 \mathrm{mg}$.The mineral premix supplied the following per kilogram of complete feed: manganese (MnSO4·H2O), $60 \mathrm{~g}$; zinc (ZnO), $40 \mathrm{mg}$; iron (FeSO4·7H2O), $80 \mathrm{mg}$; copper (CuSO4.5H2O), 8 mg; selenium (Na2SeO3), $0.2 \mathrm{mg}$; iodine (Iodized $\mathrm{NaCl}$ ), $0.8 \mathrm{mg}$; cobalt (CoCl2), $0.4 \mathrm{mg}$. 
${ }^{2}$ Calculated according to NRC (1994).

${ }^{3}$ Determined according to AOAC (2003).

Table 4. Live body weight ( $\bar{x}_{ \pm S E}$ ), $g$ of broiler chickens as affected by fiber source, levels, enzyme supplementation and their interactions

\begin{tabular}{|c|c|c|c|c|c|}
\hline \multirow{2}{*}{\multicolumn{3}{|c|}{ Item }} & \multicolumn{3}{|c|}{ Age in weeks } \\
\hline & & & 1 & 3 & 5 \\
\hline \multicolumn{3}{|c|}{ Fiber source effect } & NS & $* *$ & $* *$ \\
\hline \multicolumn{3}{|c|}{ Corn cobs (C c) } & $125.21 \pm 0.16$ & $577.94^{\mathrm{a}} \pm 15.16$ & $1184.28^{\mathrm{a}} \pm 30.74$ \\
\hline \multicolumn{3}{|c|}{ Wheat straw (W s) } & $125.48 \pm 0.15$ & $521.06^{\mathrm{b}} \pm 16.26$ & $1001.89^{b} \pm 29.12$ \\
\hline \multicolumn{3}{|c|}{ Fiber levels effect } & NS & $* *$ & $* *$ \\
\hline \multicolumn{3}{|c|}{$3 \%$} & $125.43 \pm 0.16$ & $607.00^{\mathrm{a}} \pm 13.06$ & $1213.33^{\mathrm{a}} \pm 36.71$ \\
\hline \multicolumn{3}{|l|}{$6 \%$} & $125.34 \pm 0.19$ & $543.08^{b} \pm 17.57$ & $1093.33^{\mathrm{b}} \pm 32.73$ \\
\hline \multicolumn{3}{|c|}{$9 \%$} & $125.26 \pm 0.23$ & $498.42^{\mathrm{C}} \pm 18.60$ & $972.58^{\mathrm{C}} \pm 37.03$ \\
\hline \multicolumn{3}{|c|}{ Enzyme supplementation effect (mg/kg diet) } & NS & $* *$ & $* *$ \\
\hline \multicolumn{3}{|c|}{ Without $(0)$} & $125.39 \pm 0.10$ & $503.89^{b} \pm 14.56$ & $1020.94^{b} \pm 31.27$ \\
\hline \multicolumn{3}{|c|}{250} & $125.29 \pm 0.20$ & $595.11^{\mathrm{a}} \pm 11.52$ & $1165.22^{\mathrm{a}} \pm 34.37$ \\
\hline \multicolumn{6}{|c|}{ Interactions } \\
\hline \multicolumn{3}{|c|}{ Fiber Source $\times$ Level } & NS & NS & NS \\
\hline & 3 & & $125.48 \pm 0.18$ & $632.67 \pm 15.25$ & $1304.67 \pm 40.99$ \\
\hline \multirow[t]{3}{*}{$(\mathrm{C} \mathrm{c})$} & 6 & & $125.17 \pm 0.31$ & $569.00 \pm 22.71$ & $1180.33 \pm 37.10$ \\
\hline & 9 & & $124.97 \pm 0.30$ & $532.17 \pm 23.68$ & $1067.83 \pm 31.16$ \\
\hline & 3 & & $125.37 \pm 0.29$ & $581.33 \pm 15.94$ & $1122.00 \pm 30.21$ \\
\hline \multirow[t]{2}{*}{ (W s) } & 6 & & $125.52 \pm 0.21$ & $517.17 \pm 23.94$ & $1006.33 \pm 17.63$ \\
\hline & 9 & & $125.55 \pm 0.33$ & $464.67 \pm 22.47$ & 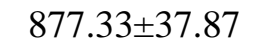 \\
\hline \multicolumn{3}{|c|}{ Fiber Source $\times$ Enzyme } & NS & NS & $* *$ \\
\hline & 0 & & $125.44 \pm 0.15$ & $532.78 \pm 17.91$ & $1103.33^{\mathrm{b}} \pm 31.24$ \\
\hline \multirow[t]{2}{*}{$(\mathrm{C} \mathrm{c})$} & 250 & & $124.97 \pm 0.25$ & $623.11 \pm 12.06$ & $1265.22^{\mathrm{a}} \pm 37.47$ \\
\hline & 0 & & $125.34 \pm 0.15$ & $475.00 \pm 19.27$ & $938.56^{\mathrm{c}} \pm 38.52$ \\
\hline \multirow{2}{*}{\multicolumn{3}{|c|}{ Fiber Level $\times$ Enzyme }} & $125.61 \pm 0.27$ & $567.11 \pm 14.92$ & $1065.22^{\mathrm{b}} \pm 33.44$ \\
\hline & & & NS & $* *$ & $* *$ \\
\hline \multicolumn{3}{|c|}{0} & $125.52 \pm 0.19$ & $573.00^{\mathrm{bc}} \pm 12.37$ & $1134.17^{\mathrm{b}} \pm 35.74$ \\
\hline \multirow[t]{2}{*}{3} & 250 & & $125.33 \pm 0.28$ & $641.00^{\mathrm{a}} \pm 11.60$ & $1292.50^{\mathrm{a}} \pm 46.32$ \\
\hline & 0 & & $125.37 \pm 0.20$ & $491.33^{\mathrm{d}} \pm 12.44$ & $1033.00^{\mathrm{b}} \pm 29.13$ \\
\hline \multirow[t]{2}{*}{6} & 250 & & $125.32 \pm 0.33$ & $594.83^{\mathrm{b}} \pm 11.48$ & $1153.67^{\mathrm{b}} \pm 49.10$ \\
\hline & 0 & & $125.30 \pm 0.16$ & $447.33^{\mathrm{e}} \pm 15.11$ & $895.67^{c} \pm 46.01$ \\
\hline 9 & 250 & & $125.22 \pm 0.45$ & $549.50^{C} \pm 15.80$ & $1049.50^{b} \pm 39.38$ \\
\hline \multicolumn{3}{|c|}{ Fiber Source $\times$ Level $\times$ Enzyme } & NS & NS & $* *$ \\
\hline & 3 & 0 & $125.50 \pm 0.29$ & $600.00 \pm 5.77$ & $1213.67^{\mathrm{c}} \pm 7.80$ \\
\hline \multirow[t]{2}{*}{$(\mathrm{C} \mathrm{c})$} & 3 & 250 & $125.47 \pm 0.29$ & $665.33 \pm 7.86$ & $1395.67^{\mathrm{a}} \pm 7.80$ \\
\hline & 6 & 0 & $125.33 \pm 0.33$ & $518.33 \pm 1.67$ & $1098.00^{\mathrm{f}} \pm 3.46$ \\
\hline$(\mathrm{C} \mathrm{c})$ & 6 & 250 & $125.00 \pm 0.58$ & $619.67 \pm 2.91$ & $1262.67^{b} \pm 9.53$ \\
\hline & 9 & 0 & $125.50 \pm 0.29$ & $480.00 \pm 7.64$ & $998.33^{\mathrm{h}} \pm 4.67$ \\
\hline$(\mathrm{C} \mathrm{c})$ & 9 & 250 & $124.43 \pm 0.30$ & $584.33 \pm 4.98$ & $1137.33^{\mathrm{e}} \pm 1.86$ \\
\hline & 3 & 0 & $125.53 \pm 0.32$ & $546.00 \pm 1.73$ & $1054.67^{g} \pm 2.60$ \\
\hline (W s) & 3 & 250 & $125.20 \pm 0.53$ & $616.67 \pm 4.41$ & $1189.33^{d} \pm 4.91$ \\
\hline & 6 & 0 & $125.40 \pm 0.31$ & $464.33 \pm 6.44$ & $968.00^{\mathrm{i}} \pm 2.31$ \\
\hline (W s) & 6 & 250 & $125.63 \pm 0.34$ & $570.00 \pm 5.77$ & $1044.67^{g} \pm 8.95$ \\
\hline & 9 & 0 & $125.10 \pm 0.10$ & $414.67 \pm 3.93$ & $793.00^{\mathrm{j}} \pm 4.62$ \\
\hline (W s) & 9 & 250 & $126.00 \pm 0.58$ & $514.67 \pm 3.18$ & $961.67^{\mathrm{i}} \pm 6.06$ \\
\hline
\end{tabular}

Means with the same letter are not significantly different $(\mathrm{P} \leq 0.05) . \quad * *=(\mathrm{P} \leq 0.01)$ and $\mathrm{NS}=$ Not significant. 
Eliwah, et al. 
Table 5. Daily body weight gain ( $\overline{\mathrm{X}}_{ \pm S E}$ ), $\mathrm{g}$ of broiler chickens as affected by fiber source, levels, enzyme supplementation and their interactions

\begin{tabular}{|c|c|c|c|c|c|}
\hline \multirow{2}{*}{\multicolumn{3}{|c|}{ Item }} & \multicolumn{3}{|c|}{ Period in weeks } \\
\hline & & & $1-3$ & $3-5$ & $1-5$ \\
\hline \multicolumn{3}{|c|}{ Fiber source effect } & $* *$ & $* *$ & $* *$ \\
\hline \multicolumn{3}{|c|}{ Corn cobs ( $\mathrm{C} \mathrm{c})$} & $32.34^{\mathrm{a}} \pm 1.08$ & $43.31^{\mathrm{a}} \pm 1.20$ & $37.82^{\mathrm{a}} \pm 1.10$ \\
\hline \multicolumn{3}{|c|}{ Wheat straw (W s) } & $28.26^{\mathrm{b}} \pm 1.16$ & $34.35^{b} \pm 1.04$ & $31.30^{\mathrm{b}} \pm 1.04$ \\
\hline \multicolumn{3}{|c|}{ Fiber levels effect } & $* *$ & $* *$ & $* *$ \\
\hline \multicolumn{3}{|c|}{$3 \%$} & $34.40^{\mathrm{a}} \pm 0.93$ & $43.31^{\mathrm{a}} \pm 1.76$ & $38.85^{\mathrm{a}} \pm 1.31$ \\
\hline \multicolumn{3}{|l|}{$6 \%$} & $29.84^{\mathrm{b}} \pm 1.26$ & $39.30^{\mathrm{b}} \pm 1.43$ & $34.57^{\mathrm{b}} \pm 1.17$ \\
\hline \multicolumn{3}{|c|}{$9 \%$} & $26.65^{\mathrm{c}} \pm 1.33$ & $33.87^{\mathrm{c}} \pm 1.46$ & $30.26^{c} \pm 1.32$ \\
\hline \multicolumn{3}{|c|}{ Enzyme supplementation effect (mg/kg diet) } & $* *$ & $* *$ & $* *$ \\
\hline \multicolumn{3}{|c|}{$\begin{array}{l}\text { Without (0) } \\
\text { lo }\end{array}$} & $27.04^{\mathrm{b}} \pm 1.04$ & $36.93^{b} \pm 1.28$ & $31.98^{\mathrm{b}} \pm 1.12$ \\
\hline \multicolumn{3}{|c|}{250} & $33.56^{\mathrm{a}} \pm 0.83$ & $40.72^{\mathrm{a}} \pm 1.68$ & $37.14^{\mathrm{a}} \pm 1.23$ \\
\hline \multicolumn{6}{|c|}{ Interactions } \\
\hline \multicolumn{3}{|c|}{ Fiber Source $\times$ Level } & $*$ & NS & NS \\
\hline \multirow{4}{*}{ (C c) } & 3 & & $36.23^{\mathrm{a}} \pm 1.09$ & $48.00 \pm 1.92$ & $42.11 \pm 1.46$ \\
\hline & 6 & & $31.70^{\mathrm{ab}} \pm 1.63$ & $43.67 \pm 1.05$ & $37.69 \pm 1.33$ \\
\hline & 9 & & $29.09^{b} \pm 1.71$ & $38.26 \pm 0.60$ & $33.67 \pm 1.12$ \\
\hline & 3 & & $32.57^{\mathrm{ab}} \pm 1.15$ & $38.62 \pm 1.06$ & $35.59 \pm 1.08$ \\
\hline \multirow[t]{2}{*}{ (W s) } & 6 & & $27.98^{\mathrm{bc}} \pm 1.71$ & $34.94 \pm 0.55$ & $31.46 \pm 0.63$ \\
\hline & 9 & & $24.22^{\mathrm{C}} \pm 1.59$ & $29.48 \pm 1.11$ & $26.85 \pm 1.35$ \\
\hline \multicolumn{3}{|c|}{ Fiber Source $\times$ Enzyme } & NS & $* *$ & $* *$ \\
\hline & 0 & & $29.10 \pm 1.28$ & $40.75^{b} \pm 1.01$ & $34.93^{b} \pm 1.12$ \\
\hline & 250 & & $35.58 \pm 0.85$ & $45.87^{\mathrm{a}} \pm 1.86$ & $40.72^{\mathrm{a}} \pm 1.33$ \\
\hline & 0 & & $24.98 \pm 1.37$ & $33.11^{c} \pm 1.53$ & $29.04^{c} \pm 1.37$ \\
\hline \multirow{2}{*}{\multicolumn{3}{|c|}{$\begin{array}{l}(\mathrm{W} \text { s) } \quad 250 \\
\text { Fiber Level } \times \text { Enzyme }\end{array}$}} & $31.54 \pm 1.07$ & $35.58^{c} \pm 1.38$ & $33.56^{\mathrm{b}} \pm 1.20$ \\
\hline & & & $* *$ & $* *$ & $* *$ \\
\hline \multirow[b]{2}{*}{3} & 0 & & $31.96^{\mathrm{bc}} \pm 0.88$ & $40.08^{b} \pm 1.69$ & $36.02^{b} \pm 1.28$ \\
\hline & 250 & & $36.83^{\mathrm{a}} \pm 0.83$ & $46.54^{\mathrm{a}} \pm 2.56$ & $41.69^{\mathrm{a}} \pm 1.65$ \\
\hline$\checkmark$ & 0 & & $26.14^{\mathrm{d}} \pm 0.89$ & $38.69^{\mathrm{b}} \pm 1.23$ & $32.42^{\mathrm{b}} \pm 1.04$ \\
\hline \multirow[t]{2}{*}{6} & 250 & & $33.54^{\mathrm{b}} \pm 0.83$ & $39.92^{b} \pm 2.71$ & $36.73^{\mathrm{b}} \pm 1.76$ \\
\hline & 0 & & $23.00^{\mathrm{e}} \pm 1.07$ & $32.02^{\mathrm{e}} \pm 2.25$ & $27.51^{\mathrm{c}} \pm 1.64$ \\
\hline \multirow{2}{*}{\multicolumn{3}{|c|}{$\begin{array}{l}9 \\
\text { Fiber Source } \times \text { Level } \times \text { Enzyme }\end{array}$}} & $30.31^{\mathrm{c}} \pm 1.15$ & $35.71^{\mathrm{bc}} \pm 1.70$ & $33.01^{\mathrm{b}} \pm 1.42$ \\
\hline & & & NS & $* *$ & $* *$ \\
\hline \multirow{3}{*}{$(\mathrm{C} \mathrm{c})$} & 3 & 0 & $33.89 \pm 0.40$ & $43.83^{c} \pm 0.49$ & $38.86^{c} \pm 0.27$ \\
\hline & 3 & 250 & $38.56 \pm 0.55$ & $52.17^{\mathrm{a}} \pm 0.88$ & $45.36^{\mathrm{a}} \pm 0.29$ \\
\hline & 6 & 0 & $28.07 \pm 0.14$ & $41.41^{\mathrm{d}} \pm 0.27$ & $34.74^{\mathrm{f}} \pm 0.12$ \\
\hline (C c) & 6 & 250 & $35.33 \pm 0.17$ & $45.93^{\mathrm{b}} \pm 0.58$ & $40.63^{\mathrm{b}} \pm 0.33$ \\
\hline & 9 & 0 & $25.32 \pm 0.54$ & $37.02^{f} \pm 0.32$ & $31.17^{\mathrm{h}} \pm 0.17$ \\
\hline (C c) & 9 & 250 & $32.85 \pm 0.36$ & $39.50^{\mathrm{e}} \pm 0.38$ & $36.18^{\mathrm{e}} \pm 0.06$ \\
\hline & 3 & 0 & $30.03 \pm 0.10$ & $36.33^{f} \pm 0.06$ & $33.18^{\mathrm{g}} \pm 0.08$ \\
\hline (W s) & 3 & 250 & $35.11 \pm 0.35$ & $40.91^{\mathrm{d}} \pm 0.60$ & $38.01^{\mathrm{c}} \pm 0.16$ \\
\hline & 6 & 0 & $24.21 \pm 0.46$ & $35.98^{f} \pm 0.31$ & $30.09^{\mathrm{i}} \pm 0.08$ \\
\hline (W s) & 6 & 250 & $31.74 \pm 0.43$ & $33.91^{\mathrm{g}} \pm 0.57$ & $32.82^{g} \pm 0.32$ \\
\hline & 9 & 0 & $20.68 \pm 0.28$ & $27.02^{\mathrm{i}} \pm 0.38$ & $23.85^{\mathrm{j}} \pm 0.17$ \\
\hline (W s) & 9 & 250 & $27.76 \pm 0.21$ & $31.93^{\mathrm{h}} \pm 0.21$ & $29.85^{\mathrm{i}} \pm 0.21$ \\
\hline
\end{tabular}


grower finisher (3 - 5 weeks of age) or overall the experimental period (1- 5 weeks of age) were significantly $(\mathrm{P} \leq 0.05)$ increased for corn cobs diets compared with wheat straw diets. It is worthy to note that, live body weight in chicks fed diets contained corn cobs as fiber source increased by 9.84 and $15.4 \%$ at 3 and 5 weeks of age respectively, when compared with those fed wheat straw diets. The corresponding figures of body weight gain were 12.61, 20.68 and $17.24 \%$ during $1-3,3-5$ and 1 - 5 weeks of age, respectively.

The reduction in growth rate (live body weight and body weight gain) as affected by wheat straw may be due to its effect on feed intake (Table 6) which resulted in a decrease in feed intake associated with increase of dietary wheat straw. Jimenez-Moreno et al. (2010) found that, body weight gain was higher $(\mathrm{P} \leq 0.01)$ for broilers fed oat hulls than for broilers fed sugar beet pulp, with those fed cellulose being intermediate.

Results of live body weight and body weight gain in the present study were in agreement with those obtained by Abou-Khashaba (1999) who, showed that the response of live body weight was directly proportional to the amount of corn cobs added, whereas broiler chicks fed the diet containing 2.5, 5 and 7.5\% corn cobs had live body weight higher than that of control group .

Hetland and Svihus (2001) pointed that birds were able to maintain adequate body weight gain when fed diet containing high levels of insoluble fiber ( $10 \%$ oat hulls), probably because fiber increase the rate of passage of the digestive system as well as the physical capacity of the gastrointestinal tract.

Results in Tables 6 and 7 revealed that, Feed intake and feed conversion were significantly $(\mathrm{P} \leq 0.01)$ affected by fiber source during the experimental periods. Using corn cobs as fiber source in broiler chick's diet significantly $(\mathrm{P} \leq 0.01)$ decreased feed intake and improved feed conversion ratio of birds during the starter, finisher and the whole experimental periods compared to wheat straw as fiber source. During the starter, finisher and the whole experimental periods, broiler fed corn cobs had lower
$(\mathrm{P} \leq 0.01)$ feed intake than broiler fed wheat straw by 2.4, 1.78 and $2.00 \%$, respectively.

Also, it is clear that groups fed corn cobs were significantly $(\mathrm{P} \leq 0.01)$ better in feed conversion ratio by $15.32,22.61$ and $19.22 \%$ than those fed wheat straw diet during the starter, finisher and the whole experimental periods, respectively.

Jimenez-Moreno et al. (2010) stated that broilers fed sugar beet pulp had lower $(\mathrm{P} \leq 0.05)$ average daily feed intake than broilers fed oat hulls, and broilers fed oat hulls, had better feed conversion ratio $(\mathrm{P} \leq 0.01)$ than broiler fed control, with those fed sugar beet pulp and cellulose being intermediate. Amerah et al. (2015) observed that, a 10\% increase in $\mathrm{AME}_{\mathrm{n}}$ intake in 21-d-old broilers fed a diet diluted with $6 \%$ cellulose with respect to those fed the control diet.

Gonzalez-Alvarado et al. (2010) reported that the inclusion in the diet of $3 \%$ SBP as a source of soluble dietary fiber reduced the average daily feed intake from 25 to 42 day of age as compared with a diet containing $3 \%$ oat hulls. However, no negative effects of sugar beet pulp inclusion were observed during the first 10 days of age.

Pettersson and Razdan (1993) reported that the inclusion of $2.3 \%$ SBP in the diet increased average daily feed intake in broilers at 14 and 21 days of age.

Muhammed et al. (2013) concluded that feed intake was not altered in broiler chick by the inclusion of two high fiber feed ingredients (DDGS or wheat bran) into the dietary formulation.

\section{Effect of fiber level}

Dietary fiber level had significant effect on live body weight at 3 and 5 weeks of age and body weight gain during all the experimental periods studied ( $1-3,3-5$ and $1-5$ weeks of age) Tables 4 and 5 . It could be noticed that, live body weight and body weight gain decreased significantly due to increase dietary fiber level in the diet from 3 to 6 or $9 \%$. The reduction in live body weight and body weight gain increased 
Table 6. Daily feed intake ( $\bar{x} \pm S E$ ), $g$ of broiler chickens as affected by fiber source, levels, enzyme supplementation and their interactions

\begin{tabular}{|c|c|c|c|c|c|}
\hline \multirow{2}{*}{\multicolumn{3}{|c|}{ Item }} & \multicolumn{3}{|c|}{ Period of weeks } \\
\hline & & & $1-3$ & $3-5$ & $1-5$ \\
\hline \multicolumn{3}{|c|}{ Fiber source effect } & $* *$ & $* *$ & $* *$ \\
\hline \multicolumn{3}{|c|}{ Corn cobs (C c) } & $63.04^{b} \pm 0.79$ & $121.95^{\mathrm{b}} \pm 0.85$ & $92.50^{\mathrm{b}} \pm 0.82$ \\
\hline \multicolumn{3}{|c|}{ Wheat straw (W s) } & $64.58^{\mathrm{a}} \pm 0.67$ & $124.17^{\mathrm{a}} \pm 0.70$ & $94.38^{\mathrm{a}} \pm 0.68$ \\
\hline \multicolumn{3}{|c|}{ Fiber levels effect } & $* *$ & $* *$ & ** \\
\hline \multicolumn{3}{|c|}{$3 \%$} & $66.87^{\mathrm{a}} \pm 0.30$ & $126.40^{\mathrm{a}} \pm 0.43$ & $96.63^{\mathrm{a}} \pm 0.36$ \\
\hline \multicolumn{3}{|l|}{$6 \%$} & $64.30^{\mathrm{b}} \pm 0.49$ & $123.63^{\mathrm{b}} \pm 0.46$ & $93.96^{b} \pm 0.47$ \\
\hline \multicolumn{3}{|l|}{$9 \%$} & $60.26^{\mathrm{c}} \pm 0.55$ & $119.16^{\mathrm{C}} \pm 0.59$ & $89.71^{\mathrm{c}} \pm 0.55$ \\
\hline \multicolumn{3}{|c|}{ Enzyme supplementation effect (mg/kg diet) } & $* *$ & $* *$ & $* *$ \\
\hline \multicolumn{3}{|c|}{ Without $(0)$} & $65.02^{\mathrm{a}} \pm 0.65$ & $124.14^{\mathrm{a}} \pm 0.79$ & $94.58^{\mathrm{a}} \pm 0.71$ \\
\hline \multicolumn{3}{|c|}{250} & $62.61^{b} \pm 0.74$ & $121.98^{b} \pm 0.78$ & $92.29^{\mathrm{b}} \pm 0.76$ \\
\hline \multicolumn{6}{|c|}{ Interactions } \\
\hline \multicolumn{3}{|c|}{ Fiber Source $\times$ Level } & $* *$ & $* *$ & $* *$ \\
\hline \multicolumn{3}{|c|}{3} & $66.41^{\mathrm{ab}} \pm 0.38$ & $125.54^{\mathrm{b}} \pm 0.48$ & $95.97^{\mathrm{ab}} \pm 0.40$ \\
\hline \multirow[t]{3}{*}{$(\mathrm{C} \mathrm{c})$} & 6 & & $63.58^{\mathrm{c}} \pm 0.67$ & $122.77^{\mathrm{C}} \pm 0.59$ & $93.18^{\mathrm{C}} \pm 0.62$ \\
\hline & 9 & & $59.14^{\mathrm{e}} \pm 0.66$ & $117.54^{\mathrm{e}} \pm 0.50$ & $88.34^{\mathrm{e}} \pm 0.55$ \\
\hline & 3 & & $67.33^{\mathrm{a}} \pm 0.41$ & $127.26^{\mathrm{a}} \pm 0.53$ & $97.30^{\mathrm{a}} \pm 0.46$ \\
\hline \multirow[t]{2}{*}{ (W s) } & 6 & & $65.02^{\mathrm{bc}} \pm 0.64$ & $124.48^{b} \pm 0.55$ & $94.75^{\mathrm{b}} \pm 0.58$ \\
\hline & 9 & & $61.38^{d} \pm 0.62$ & $120.77^{d} \pm 0.48$ & $91.08^{\mathrm{d}} \pm 0.54$ \\
\hline \multicolumn{3}{|c|}{ Fiber Source $\times$ Enzyme } & NS & NS & NS \\
\hline \multicolumn{3}{|c|}{0} & $64.26 \pm 0.99$ & $123.01 \pm 1.18$ & $93.64 \pm 1.08$ \\
\hline \multirow[t]{2}{*}{ (C c) } & 250 & & $61.83 \pm 1.14$ & $120.89 \pm 1.20$ & $91.36 \pm 1.16$ \\
\hline & 0 & & $65.77 \pm 0.82$ & $125.27 \pm 0.97$ & $95.52 \pm 0.89$ \\
\hline (W s) & 250 & & $63.39 \pm 0.94$ & $123.07 \pm 0.93$ & $93.23 \pm 0.93$ \\
\hline \multicolumn{3}{|c|}{ Fiber Level $\times$ Enzyme } & ** & NS & * \\
\hline & 0 & & $67.69^{\mathrm{a}} \pm 0.27$ & $127.43 \pm 0.46$ & $97.56^{\mathrm{a}} \pm 0.36$ \\
\hline \multirow[t]{2}{*}{3} & 250 & & $66.05^{b} \pm 0.23$ & $125.37 \pm 0.42$ & $95.71^{\mathrm{b}} \pm 0.29$ \\
\hline & 0 & & $65.75^{b} \pm 0.33$ & $124.81 \pm 0.41$ & $95.28^{\mathrm{b}} \pm 0.35$ \\
\hline \multirow[t]{2}{*}{6} & 250 & & $62.86^{\mathrm{c}} \pm 0.36$ & $122.44 \pm 0.46$ & $92.65^{\mathrm{c}} \pm 0.39$ \\
\hline & 0 & & $61.61^{\mathrm{d}} \pm 0.52$ & $120.18 \pm 0.75$ & $90.89^{d} \pm 0.61$ \\
\hline & 250 & & $58.92^{\mathrm{e}} \pm 0.57$ & $118.13 \pm 0.75$ & $88.52^{\mathrm{e}} \pm 0.64$ \\
\hline \multicolumn{3}{|c|}{ Fiber Source $\times$ Level $\times$ Enzyme } & NS & NS & NS \\
\hline & 3 & 0 & $67.19 \pm 0.27$ & $126.48 \pm 0.25$ & $96.83 \pm 0.23$ \\
\hline (C c) & 3 & 250 & $65.62 \pm 0.19$ & $124.60 \pm 0.46$ & $95.11 \pm 0.14$ \\
\hline & 6 & 0 & $65.07 \pm 0.19$ & $123.98 \pm 0.25$ & $94.52 \pm 0.09$ \\
\hline (C c) & 6 & 250 & $62.10 \pm 0.17$ & $121.57 \pm 0.48$ & $91.83 \pm 0.27$ \\
\hline & 9 & 0 & $60.52 \pm 0.37$ & $118.57 \pm 0.41$ & $89.55 \pm 0.19$ \\
\hline (C c) & 9 & 250 & $57.76 \pm 0.33$ & $116.50 \pm 0.15$ & $87.13 \pm 0.09$ \\
\hline & 3 & 0 & $68.19 \pm 0.23$ & $128.38 \pm 0.31$ & $98.29 \pm 0.23$ \\
\hline (W s) & 3 & 250 & $66.48 \pm 0.21$ & $126.14 \pm 0.27$ & $96.31 \pm 0.21$ \\
\hline & 6 & 0 & $66.43 \pm 0.21$ & $125.64 \pm 0.27$ & $96.04 \pm 0.20$ \\
\hline (W s) & 6 & 250 & $63.62 \pm 0.19$ & $123.31 \pm 0.25$ & $93.46 \pm 0.11$ \\
\hline & 9 & 0 & $62.69 \pm 0.25$ & $121.79 \pm 0.21$ & $92.24 \pm 0.02$ \\
\hline$(\mathrm{W} \mathrm{s})$ & 9 & 250 & $60.07 \pm 0.42$ & $119.76 \pm 0.31$ & $89.92 \pm 0.32$ \\
\hline
\end{tabular}


Table 7. Feed conversion ( $\overline{\mathrm{X}}_{ \pm} \mathrm{SE}$ ), $\mathrm{g}$ of broiler chickens as affected by fiber source, levels, enzyme supplementation and their interactions

\begin{tabular}{|c|c|c|c|c|c|}
\hline \multirow{2}{*}{\multicolumn{3}{|c|}{ Item }} & \multicolumn{3}{|c|}{ Period in weeks } \\
\hline & & & $1-3$ & $3-5$ & $1-5$ \\
\hline \multicolumn{3}{|c|}{ Fiber source effect } & $* *$ & $* *$ & $* *$ \\
\hline \multicolumn{3}{|c|}{ Corn cobs $(\mathrm{C} \mathrm{c})$} & $1.99^{\mathrm{b}} \pm 0.07$ & $2.84^{\mathrm{b}} \pm 0.06$ & $2.48^{\mathrm{b}} \pm 0.06$ \\
\hline \multicolumn{3}{|c|}{ Wheat straw (W s) } & $2.35^{\mathrm{a}} \pm 0.10$ & $3.67^{\mathrm{a}} \pm 0.10$ & $3.07^{\mathrm{a}} \pm 0.10$ \\
\hline \multicolumn{3}{|c|}{ Fiber levels effect } & $* *$ & $* *$ & $* *$ \\
\hline \multicolumn{3}{|c|}{$3 \%$} & $1.96^{\mathrm{c}} \pm 0.06$ & $2.97^{\mathrm{c}} \pm 0.12$ & $2.52^{\mathrm{c}} \pm 0.09$ \\
\hline \multicolumn{3}{|l|}{$6 \%$} & $2.21^{\mathrm{b}} \pm 0.11$ & $3.19^{\mathrm{b}} \pm 0.12$ & $2.76^{\mathrm{b}} \pm 0.10$ \\
\hline \multicolumn{3}{|c|}{$9 \%$} & $2.34^{\mathrm{a}} \pm 0.14$ & $3.60^{\mathrm{a}} \pm 0.18$ & $3.04^{\mathrm{a}} \pm 0.16$ \\
\hline \multicolumn{3}{|c|}{ Enzyme supplementation effect (mg/kg diet) } & $* *$ & $* *$ & $* *$ \\
\hline \multicolumn{3}{|c|}{ Without (0) } & $2.46^{\mathrm{a}} \pm 0.08$ & $3.44^{\mathrm{a}} \pm 0.13$ & $3.02^{\mathrm{a}} \pm 0.11$ \\
\hline \multirow{2}{*}{\multicolumn{3}{|c|}{$\begin{array}{l}250 \\
\text { Interactions }\end{array}$}} & $1.88^{\mathrm{b}} \pm 0.04$ & $3.08^{\mathrm{b}} \pm 0.12$ & $2.53^{b} \pm 0.08$ \\
\hline & & & & & \\
\hline & $* *$ & $* *$ & $* *$ \\
\hline \multicolumn{3}{|c|}{ Fiber Source $\times \underset{3}{\text { Level }}$} & $1.85^{\mathrm{c}} \pm 0.07$ & $2.64^{\mathrm{e}} \pm 0.11$ & $2.29^{\mathrm{d}} \pm 0.09$ \\
\hline \multirow[t]{3}{*}{ (C c) } & 6 & & $2.04^{\mathrm{bc}} \pm 0.13$ & $2.82^{\mathrm{de}} \pm 0.08$ & $2.49^{\mathrm{cd}} \pm 0.10$ \\
\hline & 9 & & $2.08^{\mathrm{bc}} \pm 0.14$ & $3.08^{\mathrm{cd}} \pm 0.06$ & $2.64^{\text {cd }} \pm 0.10$ \\
\hline & 3 & & $2.09^{\mathrm{bc}} \pm 0.08$ & $3.31^{\mathrm{bc}} \pm 0.10$ & $2.75^{\mathrm{bc}} \pm 0.10$ \\
\hline \multirow[t]{2}{*}{ (W s) } & 6 & & $2.38^{\mathrm{ab}} \pm 0.17$ & $3.57^{b} \pm 0.05$ & $3.02^{b} \pm 0.08$ \\
\hline & 9 & & $2.60^{\mathrm{a}} \pm 0.20$ & $4.13^{\mathrm{a}} \pm 0.17$ & $3.44^{\mathrm{a}} \pm 0.19$ \\
\hline \multicolumn{3}{|c|}{ Fiber Source $\times$ Enzyme } & $* *$ & NS & $* *$ \\
\hline & 0 & & $2.23^{b} \pm 0.07$ & $3.03 \pm 0.05$ & $2.70^{\mathrm{b}} \pm 0.06$ \\
\hline & 250 & & $1.74^{\mathrm{d}} \pm 0.01$ & $2.66 \pm 0.08$ & $2.26^{c} \pm 0.05$ \\
\hline & 0 & & $2.68^{\mathrm{a}} \pm 0.11$ & $3.84 \pm 0.17$ & $3.34^{\mathrm{a}} \pm 0.14$ \\
\hline \multirow{2}{*}{\multicolumn{3}{|c|}{$\begin{array}{lc}(\mathrm{W} \text { s) } & 250 \\
\text { Fiber Level } \times \text { Enzyme }\end{array}$}} & $2.02^{\mathrm{c}} \pm 0.04$ & $3.49 \pm 0.11$ & $2.80^{\mathrm{b}} \pm 0.07$ \\
\hline & & & $* *$ & $* *$ & $* *$ \\
\hline & 0 & & $2.13^{b} \pm 0.07$ & $3.21^{\mathrm{b}} \pm 0.15$ & $2.73^{\mathrm{bc}} \pm 0.11$ \\
\hline \multirow[t]{2}{*}{3} & 250 & & $1.80^{\mathrm{c}} \pm 0.05$ & $2.74^{\mathrm{b}} \pm 0.16$ & $2.32^{c} \pm 0.10$ \\
\hline & 0 & & $2.53^{\mathrm{a}} \pm 0.10$ & $3.24^{\mathrm{b}} \pm 0.11$ & $2.96^{\mathrm{b}} \pm 0.11$ \\
\hline \multirow[t]{2}{*}{6} & 250 & & $1.88^{\mathrm{bc}} \pm 0.06$ & $3.14^{\mathrm{b}} \pm 0.22$ & $2.55^{\mathrm{bc}} \pm 0.13$ \\
\hline & 0 & & $2.71^{\mathrm{a}} \pm 0.15$ & $3.86^{\mathrm{a}} \pm 0.29$ & $3.37^{\mathrm{a}} \pm 0.22$ \\
\hline 9 & 250 & & $1.96^{\mathrm{bc}} \pm 0.09$ & $3.35^{\mathrm{ab}} \pm 0.18$ & $2.71^{\mathrm{bc}} \pm 0.14$ \\
\hline \multicolumn{3}{|c|}{ Fiber Source $\times$ Level $\times$ Enzyme } & NS & $* *$ & $* *$ \\
\hline & 3 & 0 & $1.99 \pm 0.03$ & $2.88^{\mathrm{j}} \pm 0.04$ & $2.49^{\mathrm{f}} \pm 0.02$ \\
\hline \multirow[t]{2}{*}{ (C c) } & 3 & 250 & $1.70 \pm 0.02$ & $2.39^{\mathrm{i}} \pm 0.03$ & $2.10^{\mathrm{i}} \pm 0.01$ \\
\hline & 6 & 0 & $2.32 \pm 0.01$ & $2.99^{\mathrm{fj}} \pm 0.02$ & $2.72^{\mathrm{e}} \pm 0.01$ \\
\hline \multirow[t]{2}{*}{ (C c) } & 6 & 250 & $1.76 \pm 0.01$ & $2.65^{\mathrm{h}} \pm 0.04$ & $2.26^{\mathrm{h}} \pm 0.03$ \\
\hline & 9 & 0 & $2.39 \pm 0.06$ & $3.20^{\mathrm{e}} \pm 0.03$ & $2.88^{d} \pm 0.01$ \\
\hline (C c) & 9 & 250 & $1.76 \pm 0.03$ & $2.95^{\mathrm{j}} \pm 0.03$ & $2.41^{\mathrm{j}} \pm 0.01$ \\
\hline & 3 & 0 & $2.27 \pm 0.02$ & $3.53^{\mathrm{cd}} \pm 0.01$ & $2.96^{\mathrm{c}} \pm 0.01$ \\
\hline (W s) & 3 & 250 & $1.90 \pm 0.02$ & $3.09^{\mathrm{f}} \pm 0.04$ & $2.53^{\mathrm{f}} \pm 0.01$ \\
\hline & 6 & 0 & $2.75 \pm 0.06$ & $3.49^{\mathrm{d}} \pm 0.03$ & $3.19^{\mathrm{b}} \pm 0.01$ \\
\hline (W s) & 6 & 250 & $2.00 \pm 0.02$ & $3.64^{\mathrm{bc}} \pm 0.07$ & $2.85^{\mathrm{d}} \pm 0.03$ \\
\hline & 9 & 0 & $3.03 \pm 0.03$ & $4.51^{\mathrm{a}} \pm 0.06$ & $3.87^{\mathrm{a}} \pm 0.03$ \\
\hline (W s) & 9 & 250 & $2.16 \pm 0.03$ & $3.75^{b} \pm 0.03$ & $3.01^{\mathrm{c}} \pm 0.03$ \\
\hline
\end{tabular}


with increasing dietary crude fiber level from 3 to 6 or $9 \%$. At 5 weeks of age, live body weight decreased by 17.88 and $19.85 \%$, when crude fiber increased from 3 to 6 or $9 \%$ in the diet, respectively.

It is worthy mentioned that, growth performance (live body weight and body weight gain) of broiler chicks fed diet containing 3\% crude fiber were better than those fed diet contained $6 \%$ or 9\% during the all experimental periods, indicating that, the chicks could not tolerate such level. This might be due to reduction in the utilization of the basal portion in such higher fiber diet. Davorak and Bray (1978) reported that, the lowered utilization of high fiber diet may be due partly to an increase rate of passage of the feed through the digestive tract, thereby reducing the time of ingesta exposure to enzymatic degradation and the time of nutrient contact with the absorptive membranes. The inert material (fiber) may have sufficiently distended the digestive tract, thereby limiting the mixing of ingesta with digestive secretions and reducing the proportion of the total ingesta making contact with the absorptive surfaces to the extent that digestion and absorption were reduced. In addition, greater abrasive action of the added fiber may have contributed to maintenance costs for gut epithelial replacement.

Adverse effect was observed when crude fiber was increased from 3 to 6 or $9 \%$. In this concern Abbas (1992) and Aboul-Ela et al. (2005) stated that chicks fed the diet containing $7 \%$ crude fiber showed no significant in live body weight and body weight gain . Increasing crude fiber may decrease the availability of amino acid (Nwokolo et al., 1976) and almost decreased feed intake (Soliman et al., 1996; Aboul-Ela et al., 2005).

The results revealed that dietary crude fiber level had a significant $(\mathrm{P} \leq 0.01)$ effect on feed intake and feed conversion during the all studied experimental periods (1- 3, 3- 5 and 1- 5 weeks of age). It was observed that, a decrease in daily feed intake and poorest of feed conversion increased with increasing the level of dietary crude fiber from 3 to 6 or $9 \%$ with significant difference between them.

Increasing dietary crude fiber from 3 to 6 and 9\%, feed intake decreased from 9.88 to 5.73 and $7.16 \%$ during the whole experimental period, the corresponding values for feed conversion values were 16.24, 17.5 and $17.10 \%$, respectively.

Sklan et al. (2003) found that feed efficiency decreased when diets contained 80 to 90 g crude fiber $/ \mathrm{kg}$.

Mateos et al. (2012) stated that, an increase in the crude fiber content of the diet from 3 to $9 \%$, increased average daily feed intake from 1 to 4 weeks and from 11 to 14 weeks of age. An increase in dietary crude fiber hindered feed conversion ratio from 1 to 4 weeks of age.

\section{Effect of Kemzyme supplementation}

In the present study, enzyme supplementation of the experimental diets significantly $(\mathrm{P} \leq 0.01)$ improved live body weight at 3 and 5 weeks of age and body weight gain through all the experimental periods. compared with those fed unsupplemented one. This improvement was to the extent 15.33 and $12.38 \%$ for live body weight at 3 and 5 weeks of age, respectively and $19.43,9.31$ and $13.89 \%$ for body weight gain during $1-3,3-5$ and $1-5$ weeks of age, respectively. The improvement in live body weight and body weight gain due to enzyme supplementation may be attributed to feed intake (Table 6). Also, increased in digestion and absorption of all nutrients and not simply to the starch alone (Bedford and Morgan, 1996). Moreover, Non starch polysaccharides may coat the nutrients contained in the feed. The addition of cell wall degrading enzymes may release nutrients coated by non strach polysaccharides (NSP) contained in the feed and favor their digestion (Classen, 1996 and Cowan et al., 1996). It is well known that also, enzymes decrease the viscosity of the digestive contents (Bedford, 1995), which may allow a better contact of nutrients with endogenous and absorptive mucosae cells and there for a better use of the diet. Marquardt et al. (1996) observed the enzymes caused a decrease in the water content of excreta, which will benefit a management productivity and quality of the end product.

Our results disagree with those obtained by Mohamed and Hamza, 1991; Ghazalah et al., 1994) who indicated that enzyme preporation 
failed to obtain a significant increase in live body weight and body weight gain of broiler chicks.

However, other investigate found an improvement in broilers and quail chicks growth performance (live body weight and body weight gain) with enzyme supplementation of diet including high level of fiber (Zeweil, 1996; Attia and Abd El-Rahmane, 2001; Aboul Ela et al., 2005; Khan et al., 2006; Yu et al., 2007; Yang et al., 2008; Abudabos and Aljumaah, 2010; Ahsan et al., 2012; Ali et al., 2014; Amerah et al., 2015).

Results in Tables 6 and 7 revealed that, enzyme supplementation of the experimental diets rightly increased feed intake $(\mathrm{P} \leq 0.01)$ and improved feed conversion values through all the experimental periods (1-3, 3- 5 and 1- 5 weeks of age). It is worth to note that, addition of kemzyme to the diets of broiler chicks significantly $(\mathrm{P} \leq 0.01)$ enhanced the increase in feed intake by $3.70,1.74$ and $2.42 \%$ during $1-3$ , 3- 5 and 1-5 weeks of age, respectively. The corresponding improvements in feed conversion were 23.6, 10.46 and $16.22 \%$, respectively. Enzyme supplementation increases the rate of passage which may improve feed intake (Brenes et al., 1996) and decreases multiplication of anacrobes of genus clostridium (Ward, 1995). Pettersson and Aman (1989) has established that, supplementation with appropriate enzyme can partially degrade feed indosperm cell wall, giving a more rapid and extensive digestion of starch, protein and other nutrients in the small intestine, and consequently a higher feed intake and better feed conversion efficiency.

The present results are in agreement with those obtained by Zeweil (1996) who indicated that kemzyme supplementation in growing Japanese quail diets significantly improved feed consumption and feed conversion. Brenes et al. (1993) found that, addition of Roxazyme and Avizyme to diets containing bed ford barley improved feed to gain ratio by about $5 \%$ over a 6 weeks period for both male and female broilers.

Khan et al. (2006) found that birds fed the enzymes supplemented diets ate more and had better feed conversion $(\mathrm{P}<0.05)$ than those fed the control diet .

Mushtaq et al (2008) observed that enzyme supplementation during 1- 42 days decreased the feed intake and improved gain feed ratio.

Ahsan et al. (2012) revealed that broiler fed different enzymes significantly consumed 5.9 9.9\% more feed and improved $3.5-7.5 \%$ feed conversion ratio as compared with control one.

Amerah et al. (2015) observed that enzyme supplementation improved $(\mathrm{P}<0.05)$ feed conversion ratio compared with the unsupplemented diets.

Ali et al. (2014) found significant improvement on feed intake due to enzymes supplementation on broiler chick's diet.

However, other investigators indicated that, enzyme preporation failed to obtain significant improvement in feed intake or feed conversion ratio (Mohamed and Hamza, 1991; Ghazalah et al., 1994, Arce et al., 2009 ; Ali et al. 2014).

\section{Effect of interaction on fiber source $\times$ level}

There were no interaction effects between crude fiber source and level on live body weight at 3 and 5 weeks of age and body weight gain during 3- 5 and 1- 5 weeks of age (Tables 4 and 5) while, the interaction effect between fiber source and level was significant $(\mathrm{P} \leq 0.05)$ on body weight gain during 1 - 3 weeks of age (starter period). It is clear that the highest live body weight and body weight gain were recorded for chicks fed diet containing $3 \%$ crude fiber from corn cobs. On the other hand, the lowest values for live body weight and body weight gain were observed for chicks fed diet containing $9 \%$ crude fiber from wheat straw.

Within each dietary fiber source increased fiber level from 3 to 6 and 9\% significantly $(\mathrm{P} \leq 0.01)$ decreased feed intake and poorer feed conversion through the forementioned period studied. Treatment groups fed diets containing $3 \%$ crude fiber from corn cobs had significantly higher fed intake and better feed conversion during the different intervals period when compared with other treatment groups. On the other hand, chicks fed diets containing 9\% crude fiber from wheat straw had lower feed intake and poorest feed conversion. 


\section{Effect of interaction on fiber source $x$ enzyme supplementation}

Results in Tables 4 and 5 showed that, the interaction between crude fiber source and enzyme supplementation were significant $(\mathrm{P} \leq 0.01)$ on live body weight at 5 weeks of age and body weight gain through the finisher and the whole experimental periods. It is worthy to mentioned that, at any source of crude fiber, enzyme supplementation improved live body weight and body weight gain when compared to groups fed diets without enzyme supplementation.

The highest values of (live body weight and body weight gain) were recorded for chicks fed diet containing corn cobs with enzyme supplementation. While, the lowest values of (live body weight and body weight gain) were recorded by chicks fed diets containing wheat straw without enzyme supplementation.

Abou-Khashaba (1999) observed that the response of chicken's to the enzyme in live body weight was greater during the first 3 weeks of the starter period. While the beneficial effect of adding enzyme to corn cobs diet decreased by increasing the experimental corn cobs levels.

Results in Table 6 did not show any significant effects due to the interaction between fiber source and enzyme supplementation on feed intake during all the experimental periods (1-5 weeks of age) while, Results in Table 7 Show significant effect $(\mathrm{P} \leq 0.01)$ on feed conversion during 1- 3 and 1- 5 weeks of age. Within each dietary fiber source, enzyme supplementation improved feed conversion, in which the best overall mean values of feed conversion was detected for chicks fed diet containing corn cobs supplemented with kemzyme through 1- 3 and 1- 5 weeks of age . In the same respect chicks fed diet containing wheat straw without enzyme supplementation had the poorest feed conversion values.

Our results disagree with those obtained by Abou-Khashaba (1999) who showed that feed intake was significantly increased during the starter period for chicks fed on corn cobs diet supplemented with enzyme compared with control group.
Effect of interaction on fiber level $x$ enzyme supplementation

The interaction between fiber level and enzyme supplementation was significant on live body weight at 3 and 5 weeks of age and body weight gain during all the experimental periods studied (Tables 4 and 5). It could be noticed that, within each dietary fiber level, enzyme supplementation improved live body weight and body weight gain when compared with unsupplemented one. Chicks fed diet contained $3 \%$ crude fiber supplemented with enzyme recorded the highest values of live body weight at 3 and 5 weeks of age and body weight gain during all the experimental periods. While, chicks fed diet contained $9 \%$ crude fiber without enzyme supplementation recorded the lowest live body weight and body weight gain during the forementioned periods.

The interaction effect between crude fiber level and enzyme supplementation were highly significant $(\mathrm{P} \leq 0.01)$ for feed intake and feed conversion through the interval period except feed intake at 3- 5 weeks of age which, the interaction effect was not significant. The highest amount of feed intake and better feed conversion were recorded for chicks fed diet containing 3\% crude fiber without enzyme supplementation. In the same respect diet containing 9\% crude fiber without enzyme supplementation had the lowest feed intake and poorest feed conversion.

\section{Effect of interaction on fiber source $\times$ level $\times$ enzyme supplementation}

Results in Tables 4 and 5 showed that, live body weight at 5 weeks of age and body weight gain during 3- 5 and 1- 5 weeks of age .were significantly affected $(\mathrm{P} \leq 0.01)$ due to the interaction between fiber source $x$ level $x$ enzyme supplementation. Taking live body weight and body weight gain into consideration, it could be concluded that, corn cobs diets at 3\% crude fiber level with enzyme supplementation would be suitable of broiler chicks through 1- 5 weeks of age for obtained high growth rate (live body weight and body weight gain).

The interaction among fiber source $\times$ level $\times$ enzyme supplementation Tables 6 and 7 was not 
significant for feed intake and highly significant $(\mathrm{P} \leq 0.01)$ for feed conversion through 3- 5 and 15 weeks of age. Remarkably, the best overall mean values of feed conversion were recorded for chicks fed diet containing 3\% crude fiber from corn cobs and supplemented with kemzyme, while the poorest values were recorded by chicks fed corn cobs diets containing 9\% crude fiber without enzyme supplementation.

It could be concluded that, corn cobs diet at $3 \%$ crude fiber level with enzyme supplementation would be suitable for broiler chicks through out 1- 5 weks of age for obtained highe growth performance.

\section{REFERENCES}

AOAC (2003). Association of Official Analytical Chemists Official Methods of Analysis $15^{\text {th }}$ Ed. Published the AOAC. Washington DC.

Abbas, A.M. (1992). Effect of dietary fiber on broiler performance. J. Agri. Sci., Mansoura Univ., 17 (19): 3165- 3173.

Abdelsamie, R.E. and F. Yadiwilo (1981). Water consumption and effects of water restriction on performance of broilers in the tropics. Br. Poult. Sci., 22:423-429.

Abou-Khashaba, H.A. (1999). Nutritional studies on broiler rations. Ph.D. Thesis, Fac. Agric., Zagazig Univ.

Aboul-Ela, S.S., A.I. Attia, M.M. Soliman and M. Fathi (2005). Effect of sunflower meal replacement for soybean meal with / without enzyme supplementation on growing and laying performance of Japanese quail. Egyption J. Nunt. and Feeds, 8 (1): 79-103.

Abudabos, A. and R. Aljumaah (2010). Evaluation of digestible lysine needs for male broiler. Int. J. Poult. Sci., 9:1146-1151.

Acamovic, T. (2001). Commercial application of enzyme technology for poultry production In: World's Poult. Sci. J., 57: 226-242.

Ahsan, U.H., S. Ahmed, M. Yousaf, A. Rehman, M.U. Sohail and S.U. Rahman (2012). Effect of feeding whole linseed as a source of polyunsaturated fatty acids on performance and egg characteristics of laying hens kept at high ambient temperature. Brazillian J. Poult. Sci. Sept., 15 (1): 21-26.

Ali, O.A.A., N. Suthama and L.D. Mahfud (2014). The effect of feeding black cumin (Nigella sativa) and vitamin c on blood lipid profiles and growth performance of broilers. Int. Ref. J. Engin. and Sci., 3(4):28-33.

Amerah, A.M., K. Van de Belt and J.D. Van Der Klis (2015). Effect of different levels of rapeseed meal and sunflower meal and enzyme combination on the performance, digesta viscosity and carcass traits of broiler chickens fed wheat-based diets. Anim. Res. Gate, 15 (1):1-7.

Arce, M.J., E. Avila-Gonzalez, C. Lopez-Coello, L. Garibay- Torres and L.A.Martinez-Lemus (2009). Body weight, feed-particle size, and ascites incidence revisited. J. Appl. Poult. Res., 18: 465-471.

Attia, Y.A. and S.A. Abd El-Rahmane (2001). Impact of multienzymes or yea sacc supplementation on growth performance and some carcass parameters of broiler chicks fed triticale containing diets. Arch. Gefugelk., 65 (4): 168-177.

Bedford, M.R. (1995). Mechanism of action and potential environment benefits from the use of feed enzymes. Anim. Feed Sci.Technol. 53:145-155.

Bedford, M.R. and A.J. Morgan (1996). The use of enzymes in poultry diets. World's Poult. Sci. J., 52: 61-68.

Branton, S.L., B.D. Lott, J.W. Deaton, W.R. Maslin, F.W. Austin, L.M. Pote and E.J. Day (1997). The effect of added complex carbohydrates or added dietary fiber on necrotic enteritis lesions in broiler chickens. Poult. Sci., 76 (1): 24-28.

Brenes, A., R. Lazaro, M. Garcia and G.G. Mateos (1996). Utilization practica de complejos enzimaticos en aviculture. In : Rebdler, P.G., Mateos, G.G and de Blas, C. (eds) XII Curso de Especializacion FEDNA. FEDNA, Madrid, 135-157.

Brenes, A.M., M. Smith, W.G. Guenter and R.R. Marquardt (1993). Effect of enzyme supplementation on the performance and 
digestive tract size of broiler chickens fed wheat and barley based diets. Poult. Sci., 72 : 1731-1739.

Classen, H.L. (1996). Cereal grain starch and exogenous enzymes in poultry diets. Anim. Feed Sci. Technol., 62:21-27.

Cowan, W.D., A. Korsbak, T. Hastrup and P.B. Rasmussen (1996). Influence of added microbial enzymes on energy and protein availability of selected feed ingredients. Anim. Feed Sci. Technol., 60 : 311 - 319

Davorak, R.A. and D.J. Bray (1978). Influence of cellulose and ambient temperature on feed intake and growth of chicks . Poult. Sci., 57: 1351- 1354 .

Duncan, D.B. (1955). Multiple Range and Multiple F Tests. Biometrics, 11: 1-42.

Ghazalah, A.A., O.M. El-Husseiny, H.M. Fayek and S. El-Wafa (1994). Influence of enzyme preparations and growth promoters on broiler performance. The Second Scientific Conf. on Poult., Kafr El-Sheikh, Egypt, 140-165.

González-Alvarado, J.M., E. Jiménez-Moreno, D. González-Sánchez, R. Lázaro and G.G. Mateos (2010). Effect of inclusion of oat hulls and sugar beet pulp in the diet on productive performance and digestive traits of broilers from 1 to 42 days of age. Anim. Feed Sci. Technol., 162:37- 46.

González-Alvarado, J. M., E. Jiménez-Moreno, R. Lázaro and G.G. Mateos (2007). Effects of type of cereal, heat processing of the cereal, and inclusion of fiber in the diet on productive performance and digestive traits of broilers. Poult. Sci., 86:1705-1715.

Hedemann, M.S., M. Eskildsen, H.N. Laerke, C. Pedersen, J.E. Lindberg, P. Laurinen and K.E. Bach-Knudsen (2006). Intestinal morphology and enzymatic activity in newly weaned pigs fed contrasting fiber concentrations and fiber properties. J. Anim. Sci., 84:1375-1386.

Hetland, H. and B. Svihus (2001). Effect of oat hulls on performance, gut capacity and feed passage time in broiler chickens. Br. Poult. Sci., 42:354-361.
Hetland, H., B. Svihus and A. Krogdahl (2003). Effects of oat hulls and wood shavings on digestion in broilers and layers fed diets based on whole or ground wheat. Br. Poult. Sci., 44:275-282.

Hill, F.W. and L.M. Dansky (1954). Studies of the energy requirements of chickens 1-The effect of dietary energy level on growth and feed comsumption. Poult. Sci., 33: 112 - 119.

Janssen, W. M. M. A., and B. Carré. (1985). Influence of fibre on digestibility of poultry feeds. Pages 71-88 in Recent Advances in Animal Nutrition. W. Haresign and D. J. A. Cole, ed. Butterworths, London, UK.

Jiménez-Moreno, E., J.M. González-Alvarado, D. González-Sánchez, R. Lázaro and G.G. Mateos (2010). Effects of type and particle size of dietary fiber on growth performance and digestive traits of broilers from 1 to 21 days of age. Poult. Sci., 89: 2197 - 2212.

Khan, S.H., R. Sardar and B. Siddique (2006). Influence of enzymes on performance of broilers fed sunflower-corn based diets. Pak. J., 26: 109-114.

Larrea, P.M. and P.A. Garcia (1963). Growth stimulating effect of high crude fiber rations . World's Poult. Sci., 19: 282 - 288 .

Lyons, T.P. (1995). Biotechnology in the feed industry: A look forward and backward. In: Official proceedings $13^{\text {th }}$ Annual pacific Northwest Animal Conference, October 1012, Marriott Hotel, Portland, OR, USA, 4973.

Marquardt, R.R., A. Brenes, Z. Zhiqun and D. Boros (1996). Use of enzymes to improve nutrient availability in poultry feedstuffs. Anim. Feed Sci. Technol., 60: 321-330.

Mateos, G.G., E. Jimenez-Moreno, M.P. Serrno and R.P. Lazaro (2012). Poultry response to high levels of dietary fiber sources varying in physical and chemical characteristics. J. Appl. Poult. Res., 21: 156-174.

Mateos, G.G., F. Martin, M.A. Latorre, B. Vicente and R. Lazaro (2006). Inclusion of oat hulls in diets for young pigs based on cooked maize or cooked rice. Anim. Sci., 82: 57-63. 
McDonald, J.C. and G.M. Whitesides (2002). Poly (dimethylsiloxane) as a material for fabricating microfluidic devices. Accounts Chem. Res., 35 (7): 491-499.

McNab, J.M. and K.N. Boormann (2002). Poultry feedstuffs [electronic resource]: supply, composition, and nutritive value. 26 . CABI.

Michael, A.E. (2002). Enzyme use in commercial layer production. Multi-State Poult. Meeting, 14-16.

Mohamed, N.A. and A.S. Hamza (1991). Using enzyme preparation in corn-soybean meal broiler rations. Egypt. J. Anim. Prod., 28: 245.

Muhammed, W., M.F. Rothschild and M.E. Persia (2013). The effect of high and low dietary fiber diets on the performance of two lines of chickenes with divergent growth rates. Anim. Feed Sci. and Technol. J. Iowa State Univ. Ames, Iowa.

Mushtaq, T., M. Sarwar, G. Ahmad, M.A. Mirza, T. Ahmad, M. Athar, M.M.H. Mushtaq and U. Noreen (2008). Influence of prepress solvent-extracted cottonseed meal supplemented with exogenous enzyme and digestible lysine on performance, digestibility, carcass and immunity responses of broiler chickens. J. Anim. Physiol. An. N., 93: 253-262.

NRC (1994). National Research Council. Nutrient Requirements of Poultry, Nat. Acad. Sci., Washington, D.C., USA.

Nwokolo, E., D.B. Bragg and W.D. Kitts (1976). A method for estimating the mineral availability in feedstuffs. Poult. Sci., 55 (6): 2217-2221.

Pettersson, D. and P. Aman (1989). Enzyme supplementation of a poultry diet containing rye and wheat. Br. J. Nut., 62: 139-149.
Pettersson, D. and A. Razdan (1993). Effects of increasing levels of sugar-beet pulp in broiler chicken diets on nutrient digestion and serum lipids. Br. J. Nut., 70:127- 137.

Silversides, F.G. and M.R. Bedford (1999). Enzymes may improve energy and protein digestibility. Feedstuffs (Mar. 1):15-16.

Sklan, D., A. Smirnov and I. Plavnik (2003). The effect of dietary fiber on the small intestines and apparent digestion in the turky. Br. Poult. Sci., 44 (5):735-740.

Snedecor, G. W. and Cochran, W. G. (1982). Statistical Methods. $6^{\text {th }}$ Ed., Iowa State Univ. Press, Ames, USA. Pages: 593.

Soliman, A.Z., I. Hassan, S. Abou-Elwafa and A.G. Abdellah (1996). Utilization of high fiber sunflower meal with/without commercial enzymes of stabilized rumen extract in broiler diets. Egypt. Poult. Sci., 16 (1): 51-67.

SPSS (1999). Statistical software package for the social sciences. SPSS, Int., USA.

Tasaki, I. and V. Kibe (1959). A study on the digestion of cellulose in poultry. Poult. Sci., 38: 376-379.

Ward, N.E. (1995). With dietary modifications, wheat can be used for poultry. Feedstuffs 7 Aug, 14-16.

Yang, J.Q., J.H. Chen and J.J. Liu (2008). A new genus and a new species of Braconinae (Hymenoptera, Braconidae) from China. Acta Zootaxonomica Sinica, 33 (1): 61-64.

Yu, B., S.T. Wu, C.C. Liu, R. Gauthier and P.W.S. Chiou (2007). Effects of enzyme inclusion in a corn-soybean diet on broiler performance. Anim. Feed Sci. Technol., 134: 283-294.

Zeweil, H.S. (1996). Evaluation of substituting Nigella sativa oil meal for Soybean meal on the performance of growing and laying Japanese quails. Egypt. Poult. Sci., 16 (11): 451-477. 
تأثير مصـــدر ومســـتوى الأليــاف مـــع أو بــدون إضــافة الأنزيـم على أداء النمـو لبداري التسمين

$$
\begin{aligned}
& \text { أحمد عليوه محمد عليوه ـ صلاح الدين سيد أبو العلا ـ عادل إبراهيم عطيه ـ إسماعيل السيد إسماعيل }
\end{aligned}
$$

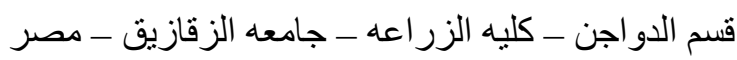

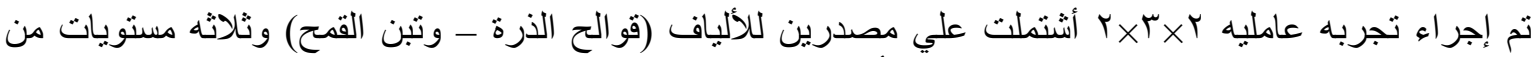

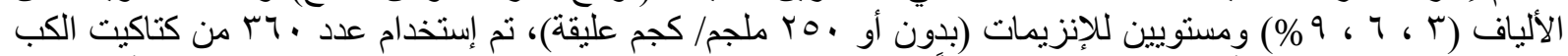

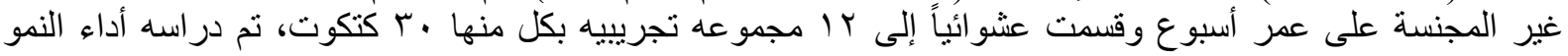

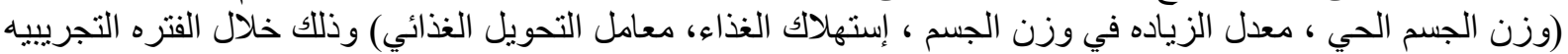

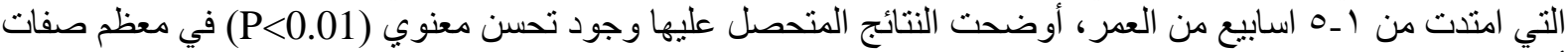

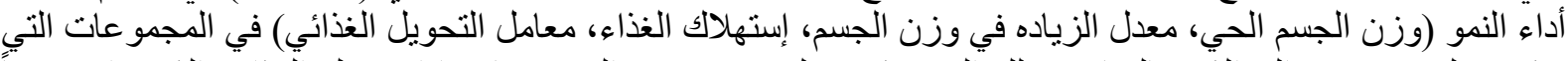

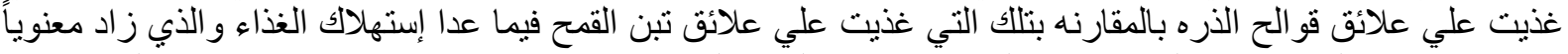
(P<0.01)

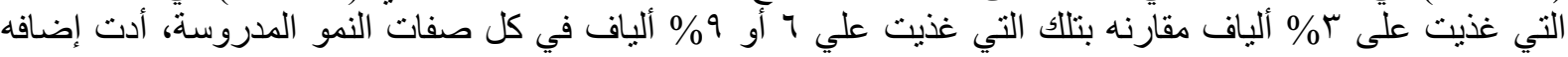

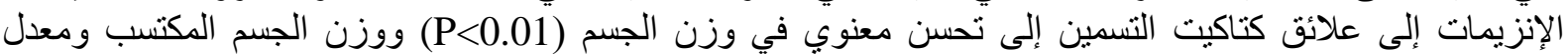

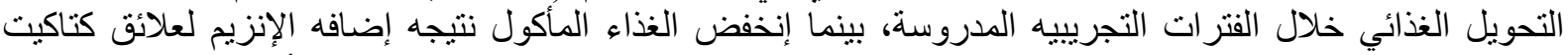

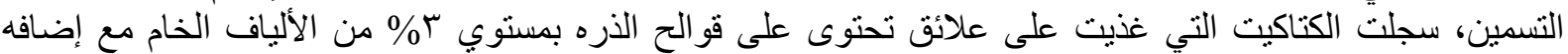

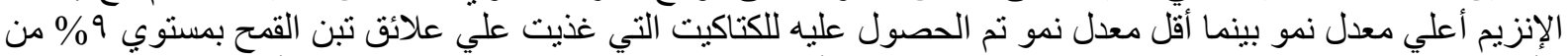

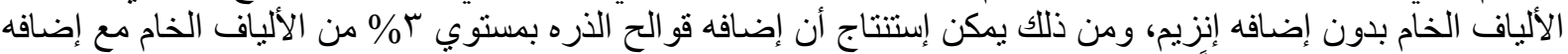
الإنزيم يمكن أن يكون مناسباً لكتاكيت التنسين خلال الفتره العمريه من ألـ الـ أسابيع.

أستاذ تغذية الدو اجن - كلية الزر اعة - جامعة القاهرة. أستاذ تغذية الدو اجن المتفرغ ـ كلية الزية الزر اعة - جامعة الزقازيق. 